\title{
La politique territoriale
}

\author{
Marc-Urbain Proulx \\ Universit du Qu bec Chicoutimi
}

\section{Introduction}

La politique gouvernementale à l'égard des régions, des villes, de la ruralité, des métropoles, des municipalités et des localités nous concerne tous directement. Par l'entremise de la réforme des agglomérations urbaines, le Québec vit actuellement l'un des moments historiques les plus intenses de sa politique territoriale, qui inclut aussi un important programme pour les régions-ressources, des incitatifs pour les cités et technopoles (agroalimentaire, multimédia, optique, aluminium...) ainsi que des mesures appliquées à la ruralité, à la forêt, aux transports, etc. Au contraire de la politique à l'égard de la santé, de la culture ou d'autres secteurs, la politique territoriale s'avère fondamentalement horizontale, multisectorielle. Elle touche à la fois les mines, l'hydroélectricité, l'éducation, l'agriculture, les diverses industries, etc. Elle interpelle donc plusieurs ministères. Elle s'inscrit dans une logique tout à fait particulière s'appliquant à des situations spécifiques, fort diverses sur l'espace québécois, même si les $616000 \mathrm{~km}^{2}$ habités sont relativement homogènes. Rien ne ressemble moins à une ville qu'une autre ville. Rien ne ressemble moins à une MRC qu'une autre MRC. Rien ne ressemble moins à une région qu'une autre région. Rien ne ressemble moins à une agglomération qu'une autre agglomération. Ce qui nécessite des interventions publiques flexibles et modulées à l'égard des territoires qu'il faut soit aménager, gérer, reconvertir, décongestionner, diversifier, revitaliser, développer ou tout cela à la fois, dans un esprit de durabilité, évidemment.

Nous avançons dans ce texte que la consolidation supralocale recherchée par la politique territoriale du Québec doit actuellement progresser sur chaque territoire en s'appuyant sur une procédure appropriée de planification globale qui sera capable d'impliquer non seulement les toutes récentes structures municipales et leurs nouveaux élus mais aussi tous les partenaires territoriaux du domaine public non municipal, du vaste domaine communautaire (société civile organisée) et les acteurs de l'ensemble des champs d'activité du secteur privé. Du coup, le processus collectif d'apprentissage à l'innovation et au développement en sera stimulé sur les divers territoires.

\section{Retrait partiel de la politique keynésienne}

D'inspiration keynésienne, la politique territoriale fut, dans ses décennies de gloire, soit de 1945 à 1985, généralement de nature macro-économique. Justifiée notamment par la loi des avantages comparés, le modèle du développement par étapes et la théorie de la localisation industrielle, elle fut très concernée par des investissements publics massifs dans la construction d'infrastructures de transport et d'équipements publics afin d'assurer la profitabilité des implantations industrielles sur des territoires peu ou aucunement exploités mais à fort potentiel. On visait le démarrage économique de ces lieux en misant sur le principe des « industries industrialisantes », celles-ci étant situées au Québec en amont des filières de production. On visait aussi l'allégement des différences interterritoriales en matière d'emplois, de revenus, de consommation, de services publics, etc.

L'aménagement des territoires fut alors la grande stratégie d'occupation et de développement du Québec central et périphérique. Dans la pure tradition macroéconomique, on a aussi alloué généreusement des droits d'exploitation de bassins de ressources naturelles alors que les allégements fiscaux devinrent la norme à l'égard des entreprises. On a largement distribué des subventions et des incitatifs à l'investisse- 
ment dans les équipements industriels. Aussi, l'État s'est approprié certains moyens de production, notamment dans les secteurs de l'hydroélectricité et de l'amiante. En outre, nos gouvernements successifs ont modulé les règles d'attribution des allocations au chômage afin d'avantager les travailleurs de certains lieux spécifiques, confrontés au travail saisonnier. Ces dernières mesures s'ajoutaient à plusieurs mesures sociales universelles qui, indirectement, stimulaient la demande et favorisaient ainsi les activités économiques dans les zones désignées en fonction de leurs difficultés économiques.

De 1945 à 1985, années pendant lesquelles la politique territoriale était d'inspiration keynésienne, on visait le démarrage économique des territoires peu ou aucunement exploités mais à fort potentiel en misant sur le principe des « industries industrialisantes », celles-ci étant situées au Québec en amont des filières de production. On visait aussi l'allégement des différences interterritoriales en matière d'emplois, de revenus, de consommation, etc.

Cette politique keynésienne fut confrontée sur deux fronts à la fin des années 1970, notamment au Québec et au Canada. On constata d'abord qu'elle n' atteignait pas totalement ses objectifs économiques par l'entremise de ses interventions de haut en bas, aussi nommées «top down». Plusieurs territoires économiquement démarrés ici et là demeuraient en effet fortement dépendants des interventions externes pour se maintenir en croissance alors qu'ailleurs, les disparités économiques et sociales demeuraient importantes à travers les territoires. On a aussi constaté que les pôles de croissance dispersés sur l'espace québécois ne diffusaient pas autant que prévu, tout en drainant néanmoins les ressources de la périphérie. Même plus, certains de ces pôles au Québec périclitaient ou stagnaient malgré des efforts importants de relance économique par l'entremise d'investissements publics et de mesures gouvernementales visant à attirer les activités privées. Aussi, la planification régionale effectuée périodiquement depuis l'exercice initiateur du BAEQ (Bureau d'aménagement de l'est du
Québec) n'a à l'évidence pas généré les instruments territoriaux nécessaires pour faire maturer les économies régionales par la diversification. Bref, l'efficacité de la politique territoriale fut questionnée, par comparaison entre les moyens consentis et les résultats obtenus.

Elle le fut par ailleurs à une époque de questionnement profond du rôle de l'État, à la faveur d'un vaste mouvement sociopolitique s'appuyant sur la doctrine néolibérale. La conjoncture fut favorable à l'écho de cette doctrine pendant la décennie 1980 par l'entremise de trois tendances de fond : l'accélération de la globalisation des échanges sous l'influence des nouvelles technologies de communication, la crise des finances publiques dans plusieurs pays à tendance social-démocrate et le démantèlement de plusieurs régimes socialistes en Europe de l'Est.

\section{Les limites du néolibéralisme}

Dans un tel contexte, la plupart des pays occidentaux ont en l'occurrence tenté d'améliorer leur productivité intérieure par un nouvel ordre macro-économique national moins empreint d'interventionnisme. La privatisation d'entreprises publiques, la déréglementation des activités économiques, la réduction de certains coûts sociaux, l'allégement des tracasseries bureaucratiques et l'amélioration de la performance des infrastructures, des équipements et des services publics furent claironnés vivement avant d'être inscrits à l'agenda politique. La mondialisation fut alors un excellent bouc émissaire de ce retrait partiel de l'État dans son rôle de régulation socio-économique. Il y eut certes en conséquence quelques progrès perceptibles dans les unités de production. Mais la plupart des spécialistes $^{1}$ constatent que les rendements d'une telle politique publique furent rapidement neutres ou décroissants en matière de productivité. En outre, les coûts associés à ces gains limités sont vite apparus importants, notamment sous l'angle des effets sociaux et environnementaux bien sûr, mais aussi sur les plans économique et politique.

En réalité, le néolibéralisme, qui était hier encore enchanteur, est désormais confronté à ses limites. D'autant plus que l'échelle mondiale à laquelle on se réfère pour se soustraire des politiques nationales n'offre que très peu de prises réelles pour influencer les conditions de la productivité. Bien sûr que l'on peut 
améliorer la mobilité des facteurs (capital et travail) à cette échelle. Bien sûr que certaines règles mondiales positives sont devenues opérationnelles, notamment la protection de la propriété intellectuelle. Mais force est de constater que l'échelle mondiale alimente peu pour le moment la régulation socio-économique pourtant nécessaire pour faire face aux fluctuations conjoncturelles et aux bouleversements structurels. Ce qui explique en grande partie le mouvement actuel marqué vers un retour à la responsabilisation de l'État dans ses interventions publiques. Et dans cette nouvelle volonté de régulation étatique, les échelles territoriales (locale, urbaine, agglomération, régionale) offrent plusieurs vertus. Elles permettent notamment à l'État de renforcer son rôle national par la régulation territoriale $^{2}$ tout en demeurant conforme aux conditions imposées par les ententes internationales de libreéchange.

Force est de constater que l'échelle mondiale alimente peu pour le moment la régulation socioéconomique pourtant nécessaire pour faire face aux fluctuations conjoncturelles et aux bouleversements structurels. Ce qui explique en grande partie le mouvement actuel marqué vers un retour à la responsabilisation de l'État dans ses interventions publiques.

\section{Le virage micro-économique}

Selon un regard international très actuel, la politique territoriale s'avère bien vivace malgré les coupures budgétaires drastiques dans certains pays tels que l'Australie, le Canada et la Russie, ou la perte de popularité d'autres pays tels que les États-Unis, l'Argentine et l'Algérie. Elle reprend même du gallon dans plusieurs pays, notamment dans de nombreux États africains et asiatiques qui vivent désormais à l'heure de la décentralisation. Il existe en outre des politiques territoriales vigoureuses au Japon en désir de déconcentration des grands centres, en Norvège afin d'occuper l'espace national, au Brésil pour exploiter les bassins périphériques de ressources, en Irlande selon une finalité d'équité spatiale, et dans bien d'autres pays comme l'Indonésie, le Mexique, le Viêtnam. Même la Chine investit massivement dans sa politique territoriale afin de diffuser vers l'intérieur la forte croissance de ses dragons côtiers tels que Beijing, Tanjin, Shanghai, Hong-Kong et Canton. Il est pertinent de noter finalement que la Communauté européenne consacre plus du tiers de son budget à cette politique, ce qui nécessite la dotation d'une vision et d'une stratégie territoriales par les pays membres, réceptacles des subsides.

L'OCDE (Organisation de coopération et de développement économique) possède d'ailleurs tout un programme de recherche à cet effet de stratégie territoriale et fournit des idées en quantité et en qualité pour définir les instruments d'intervention. L'approche n'est pas exclusivement macro-économique. Loin s'en faut. On observe en effet depuis le milieu des années 1980 une croissante implication gouvernementale dans une politique territoriale de nature micro-économique. Il semble que l'État postkeynésien désire désormais se rapprocher des agents économiques tels que les entrepreneurs, les travailleurs, les investisseurs et les consommateurs.

C'est ainsi que le soutien direct à l'entrepreneurship, aux PME et aux travailleurs indépendants obtient une part budgétaire jamais atteinte auparavant dans la politique économique. La qualité de ce soutien s'est en outre accrue rapidement à la faveur de l'expérience acquise des fabricants de mesures gouvernementales. On a à cet effet expérimenté de nouveaux outils reliés à l'incubation industrielle, au financement du risque, à la planification du démarrage d'affaires, au transfert de technologies et à l'animation économique. Ces outils sont dans la réalité presque tous ancrés sur les territoires locaux et cherchent à améliorer la qualité entrepreneuriale de l'environnement immédiat, soit le milieu, notamment au Québec avec les centres d'aide aux entreprises, les SOLIDE (sociétés locales d'investissement et de développement économique), les centres de R\&D reliés aux cégeps, les SADC (sociétés d'aide au développement des collectivités) et les sociétés de développement. Le dernier né de ces outils a pris la forme de CLD (centres locaux de développement), véritables guichets multiservices pour les activités productives de diverses natures, y compris celles du communautaire et de l'économie sociale.

Si l'échelle territoriale, locale ou supralocale devient le point de chute de la politique économique concernée par les demandeurs de travail que sont les 
entreprises, on constate cette même tendance du côté de la politique concernant les offreurs de travail que sont les travailleurs. En réalité, la régulation du marché du travail par la politique publique s'effectue de plus en plus à l'échelle des «bassins d'emplois », c'est-à-dire qu'un grand nombre de mesures concernées par la réinsertion au travail, l'amélioration de l'employabilité des ressources humaines, la formation professionnelle, les stages en entreprises, la recherche d'emplois sont désormais décentralisées à un certain degré, à l'échelle du milieu immédiat des travailleurs, défini généralement par les statistiques sur les migrations alternantes quotidiennes (navettage) entre le domicile et le travail. Bref, le soutien public aux travailleurs indiqué par la théorie du capital humain, qui domine actuellement la théorie du développement, s'effectue à l'intérieur de la politique territoriale ou du moins, par une politique sectorielle territorialisée, ce qui revient au même. Les CLE (centres locaux d'emploi) représentent au Québec l'agence territoriale pour l'application de cette politique économique.

Nous aimerions souligner quatre points importants à propos de ce nouvel enjeu de la politique publique à l'égard de l'environnement micro-économique. Ils convergent pour revitaliser le vaste domaine public territorial à l'échelle supralocale.

\section{Le domaine public supralocal en effervescence}

Notre premier point concerne le fait que les nouvelles interventions territoriales à l'égard des entreprises et des travailleurs s'inscrivent dans un vaste domaine public local déjà bien en place, reconnu démocratiquement dans la plupart des pays. Il possède une très forte spécificité en regard de la gestion de biens et services collectifs à la population. À cet effet au Québec, nos traditionnelles municipalités de diverses tailles relèvent des responsabilités plus ou moins nombreuses. Dans ce champ de consommation collective typiquement local, elles sont secondées par les commissions scolaires, les CLSC, les cégeps, les centres hospitaliers et bien d'autres centres de services, municipalisés (loisirs, communautaires, etc.) ou non (personnes âgées, handicapées, etc.). De plus, la société civile s'avère bien organisée à cette échelle par l'entremise de groupes sociaux, de groupes d'intérêt, de groupes de services, de coopératives et de diverses unions patronales et ouvrières qui sont de plus en plus sollicitées dans la desserte de services, notamment par l'entremise de l'économie sociale et de divers partenariats public - privé. En réalité, nous faisons face globalement à un vaste domaine public local fragmenté dans ses fonctions et éclaté dans son pouvoir au sein d'une panoplie d'organisations plus ou moins autonomes. Toute analyse territoriale se voit confrontée à ce contexte institutionnel.

L'enjeu à l'égard des interventions gouvernementales sur les territoires s'avère très bien justifié, et soutenu par les spécialistes du développement, notamment ceux de l'économie urbaine et régionale, de la géographie économique et l'économie publique locale. Nous vous ferons grâce ici d'une synthèse de la littérature extrêmement riche qui, depuis deux décennies, s'articule autour des concepts de développement endogène, de district industriel, de développement local, de milieu innovateur, de systèmes territoriaux d'innovation et de production, de communautés apprenantes et autres «learning regions ». Soulignons tout simplement que le mouvement très actif de recherche permet d'observer les expériences en cours, de modéliser les meilleures pratiques et d'enrichir les modèles théoriques qui offrent un cadre solide aux fabricants de politiques et de mesures micro-économiques. L'objectif principal de ce cadre est de saisir et d'influencer la création d'économies externes et d'économies de proximité. En analysant le progrès scientifique réalisé depuis vingt ans, force est de constater que cette nouvelle collaboration entre recherche et action a généré de part et d'autre des résultats fort intéressants. On comprend de mieux en mieux la réalité du soutien territorial à la production et à l'innovation. Et on influence positivement cette réalité vers le changement. Le deuxième rendez-vous mondial sur les systèmes productifs locaux, qui se tiendra à la Cité des Sciences et de l'Industrie de La Villette du 28 au 30 janvier 2002 , illustre bien cette collaboration fructueuse.

Nous faisons face globalement à un vaste domaine public local fragmenté dans ses fonctions et éclaté dans son pouvoir au sein d'une panoplie d'organisations plus ou moins autonomes. Toute analyse territoriale se voit confrontée à ce contexte institutionnel. 
Même s'il n'existe pas encore de véritable théorie bien articulée sur les systèmes productifs locaux, quelques jalons théoriques sont largement acceptés. Ainsi, le fait que l'on admette le rôle crucial de l'entrepreneur s'avère désormais un acquis. Cette ressource humaine rare est reconnue comme le porteur du risque, de l'initiative innovatrice. Dans sa quête d'intrants, il est branché sur l'univers bien sûr, mais aussi sur son milieu immédiat. La formation professionnelle, l'aide à la planification des affaires, le financement du risque, le soutien à la $R \& D$ et l'animation économique sont aussi des composantes estimées essentielles à l'activation de l'innovation et de la production sur un territoire. Mais le cœur du questionnement à propos des systèmes locaux de production concerne le processus d'apprentissage collectif alimenté par l'interaction et l'information. Cet apprentissage n'est pas simple à produire par des interventions publiques. Il n'est pas simple à mesurer non plus. Mais il peut être influencé positivement selon les potentialités de chaque territoire.

Finalement, nous constatons que cet enjeu de la politique publique à l'égard de l'environnement microéconomique permet d'établir une convergence entre les objectifs sociaux et les objectifs économiques. Les mesures actives à l'égard des travailleurs, notamment des travailleurs indépendants, en sont un excellent exemple. Le soutien à l'économie sociale permet aussi ces convergences. Et que dire de toutes ces PME qui, tout en bénéficiant de certains programmes adaptés aux besoins différents selon les lieux, créent de l'emploi tout à fait apprécié ? En réalité, l'échelle territoriale supralocale, qui au Québec est largement représentée par les MRC et les agglomérations, s'avère l'arène de nouveaux éléments positifs dans la régulation socio-économique.

Le cour du questionnement à propos des systèmes locaux de production concerne le processus d'apprentissage collectif alimenté par l'interaction et l'information. Cet apprentissage n'est pas simple à produire par des interventions publiques. Il n'est pas simple à mesurer non plus. Mais il peut être influencé positivement selon les potentialités de chaque territoire.

\section{La planification territoriale globale}

En regard de cette effervescence dans le domaine public supralocal, on constate que le Québec a expérimenté plusieurs procédures de planification au cours des dernières années. D'abord, les conseils des MRC et des communautés métropolitaines se sont dotés d'un schéma d'aménagement du territoire. Plusieurs y ont inclus une dimension développement alors que d'autres ont opté pour un plan de développement indépendant. Les SADC possèdent leur plan. Les CLD planifient aussi, en incluant la dimension de l'emploi afin d'en aviser les CLE. Les CLSC planifient la desserte de leurs services à l'échelle des MRC. Les SOLIDE élaborent leur stratégie pour leur propre territoire MRC d'intervention. Il en est de même pour les organisations qui, dans la gestion des déchets, le tourisme ou encore la sécurité publique notamment, oeuvrent à l'échelle territoriale MRC ou à celle des agglomérations. À tous ces planificateurs, il faut ajouter les municipalités locales, avec leur plan d'urbanisme et leurs plans sectoriels, et les nouvelles autorités publiques qui prennent assises formellement à l'échelle des agglomérations urbaines.

La cohérence du vaste domaine public supralocal du Québec ne peut être envisagée de la même manière que dans les activités sectorielles du vaste domaine public national largement structuré par la hiérarchie. À cette échelle supralocale, l'éclatement du pouvoir représente une donnée fondamentale, incontournable.

En réalité, de nombreux planificateurs autonomes mais interdépendants élaborent leur vision, leurs stratégies, leurs tactiques et leurs actions à l'échelle territoriale supralocale. D'aucuns suggéreront qu'il faut tout de même de la cohérence entre tous ces plans, dans un esprit de soutien au fameux processus collectif d'apprentissage.

Or, la cohérence du vaste domaine public supralocal du Québec ne peut être envisagée de la même manière que dans les activités sectorielles du vaste domaine public national largement structuré par la hiérarchie. À cette échelle supralocale, l'éclatement du pouvoir représente une donnée fondamentale, incontournable. 
Même si quelques structures sont intégrées, le pouvoir territorial demeurera éclaté. D'ailleurs, cet éclatement représente une condition nécessaire à l'efficacité administrative. Il existe bien sûr de la concurrence pour l'obtention de ressources, quelquefois exacerbée. Il y a aussi certains chevauchements de missions. Mais il existe surtout des champs spécifiques d'intervention sectorielle relativement étanches (éducation, sécurité publique, santé, loisirs, développement économique, etc.) à l'intérieur desquels chaque organisation œuvre de façon optimale et innovatrice selon sa mission proprement délimitée. Les interdépendances, les complémentarités et les convergences potentielles dans un esprit de synergie nécessitent une vision territoriale commune, partagée par tous, ainsi que des mécanismes institutionnels de collaboration. Tous en conviennent. Il est là le véritable enjeu. D'ailleurs, plusieurs territoires MRC étaient fort avancés ${ }^{3}$ sur ce chemin de la vision globale et des coalitions dans l'action avant l'arrivée de la politique de renforcement des agglomérations. Nul doute que ce cheminement progressif va se poursuivre.

Les interdépendances, les complémentarités et les convergences potentielles dans un esprit de synergie nécessitent une vision territoriale commune, partagée par tous, ainsi que des mécanismes institutionnels de collaboration.

\section{Processus d'organisation territoriale}

Le développement territorial à succès possède toujours des modalités organisationnelles particulièrement optimales ${ }^{4}$. Voilà une vérité largement induite de l'expérience récente dans les pays développés et qui, au fil des résultats de recherche, s'inscrit progressivement telle une véritable loi scientifique en théorie du développement. Diverses expressions sont utilisées dans la littérature scientifique pour désigner, saisir et modéliser ces modalités. Elles font toutes référence à la dynamique de nature collective. De ce constat, on recommande généralement au pouvoir public d'agir sur les relations entre les différents acteurs qui, dans un processus continu d'apprentissage, participent à l'organisation de leur territoire dans un esprit de progrès et de développement.
La politique urbaine, locale et régionale de plusieurs pays focalise actuellement sur cet enjeu crucial. De l'aménagement du territoire qui fut jadis au coeur des efforts publics de développement, au management territorial qui a atteint son apogée pendant les années 1970 et 1980, nous passons maintenant aux interventions sur les processus continus d'organisation territoriale ${ }^{5}$.

\section{Figure 1. Processus continu d'organisation territoriale}

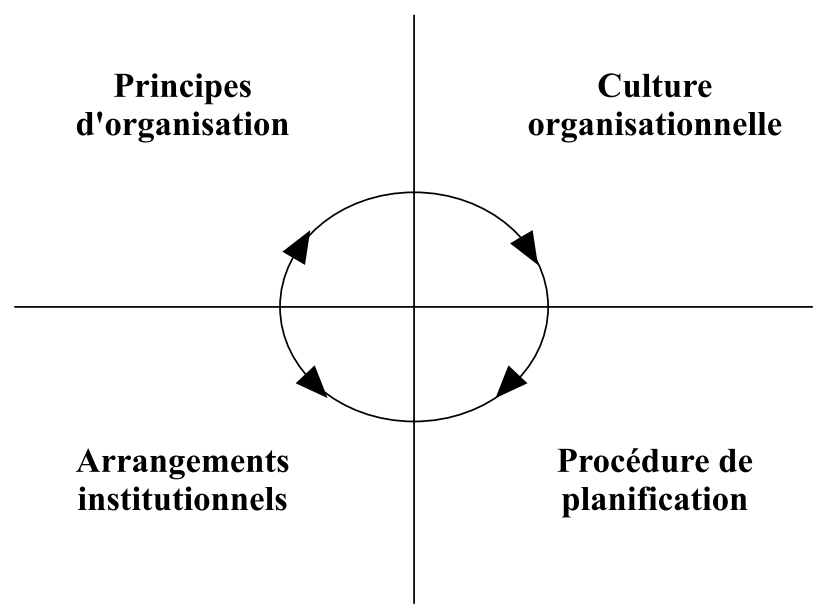

Selon notre analyse ${ }^{6}$, cette organisation collective et progressive des territoires se résume essentiellement à quatre grandes dimensions illustrées par le schéma : les principes d'organisation territoriale, la culture organisationnelle territoriale, la procédure de planification territoriale, les arrangements institutionnels optimaux. Cette dernière dimension devient l'aboutissement et aussi le départ répétitif du processus cumulatif conduisant, par surplus d'organisation, au développement territorial.

À l'échelle supralocale du Québec, nul doute que les acteurs présents ont tout intérêt à se commettre à un degré élevé dans un tel processus d'organisation territoriale. La mise en oeuvre actuelle de la réforme des agglomérations et ses conséquences rendent d'ailleurs cet engagement tout à fait impératif. Cet engagement devrait à notre avis être stimulé et soutenu formellement par une politique publique explicite. Pour ce faire, le meilleur enclencheur réside sûrement dans un exercice de planification territoriale globale de nature 
interactive, incluant l'aménagement du territoire, la gestion publique de biens et services collectifs et la promotion du développement social, culturel et économique.

\section{Notes et références}

1 Voir PORTER, M. E. (2001). «Regions and the New Economics of Competition », in A. J. Scott, Global CityRegions, Oxford University Press, New York, p. 139-157.

2 Voir PROULX, M.-U. (1999). «Le rôle des territoires dans la régulation de l'économie-monde ", dans M.-U. Proulx (éd.), Territoires et Développement économique, Éditions L'Harmattan, Paris.
3 Voir JEAN, N. et M.-U. PROULX (2001). «La dynamique organisationnelle des territoires MRC », Cahiers de géographie du Québec, vol. 45, no 124, p. 87-100.

4 Voir BLAKELY, E. J. (1994). Planning Local Economic Development, Sage Publications, London, 356 p.

5 Voir GUIGOU, J.-L. (1996). L'aménagement du territoire de l'an 2000, no 3 ; VELTZ, P. (1996). Mondialisation, villes et territoires, Éditions PUF, Paris, 255 p. ; MARKUSEN, A. (1996). "Interaction between Regional and Industrial Policies », International Regional Science Review, vol. 19, nos 1-2, p. 49-78.

6 PROULX, M.-U. (1998). «L'organisation des territoires au Québec », dossier de la revue Action nationale, vol. 88, nos 2-3. 